\title{
A uniform approximation theorem and its application to moment problems
}

\author{
By \\ A. JAKIMOVSKI and M. S. RAMANUJAN
}

\section{Introduction}

The most familiar of the moment problems is the one due to HAUSDORFF [4]. It consists in determining a set of necessary and sufficient conditions in order that a (real) sequence $\mu_{n}$ may have the representation $\mu_{n}=\int_{0}^{1} t^{n} \mathrm{~d} \chi(t)$, where $\chi(t)$ is a function of bounded variation in [0,1]. HARDY [3] outlines the proof of HAUSDORFF; several alternate proofs of the result of HAUSDORFF are known (see for instance WIDDER [14] and LORENTZ [8]). One of these alternate proofs makes use of the uniform approximation of functions continuous in $[0,1]$ by their Bernstein polynomials and is, to a large extent, due to HildEBrandt [5]. His proof is what is outlined by LoRENTz. An alternate set of necessary and sufficient conditions for the same representation has been given by RAMANUJAN [10] in his investigation on the quasi-Hausdorff methods. The results of HAUSDORFF and RAMANUJAN have been generalised by JAKIMOVSKI [6], [7]. Also, the uniform approximation through the Bernstein polynomials enabled LORENTz to determine the solution of the moment problem in the function spaces of the Köthe-Toeplitz type; for the same function spaces, alternate solutions of the moment problems of LORENTZ have been provided by RAMANUJAN [12] and his solutions make use of the uniform approximation of continuous functions in $[0,1]$ by certain power series, a result demonstrated by MEYER-KöNIG and ZELLER [9].

Among the modifications of the moment problem, the one concerning the representation of $\mu_{n}$ in the form $\mu_{n}=\int_{0}^{1} t^{\lambda_{n}} d \chi(t)$, where $0=\lambda_{0}<\lambda_{1}<\cdots<\lambda_{n} \cdots$ and $\sum 1 / \lambda_{n}$ diverges and $\chi(t)$ is a function of bounded variation in $[0,1]$ is due to HAusdorfF himself. For an account of this, see SHOHAT and TAMARKin [13]. Recently ENDL [2] and JAKIMOVSKI [7] were led to consider the representation $\mu_{n}=\int_{0}^{1} t^{n+\alpha} d \chi(t)$, where $\alpha$ is real and $\chi(t)$ is, as before, a function of bounded variation. Evidently this case is not covered by HAUSDORFF's results.

They were led to this problem while considering the regularity conditions governing the summability matrix $\left(H^{\alpha}, \mu\right) \equiv\left(h_{m n}^{\alpha}\right)$, where

$$
h_{m n}^{\alpha}=\left(\begin{array}{c}
m+\alpha \\
m-n
\end{array}\right) \Delta^{m-n} \mu_{n}, \quad(m \geqq n), \quad \text { and } \quad=0, \quad(m<n),
$$


and they proved that

$$
\sup _{m} \sum_{n=0}^{m}\left|\left(\begin{array}{c}
m+\alpha \\
m-n
\end{array}\right) \Delta^{m-n} \mu_{n}\right|<\infty
$$

is a necessary and sufficient condition for the above representation of $\mu_{n}$. A close analysis of these two proofs reveals apparently no common ground, except the final result itself.

In this paper we prove a uniform approximation theorem similar to the Bernstein theorem and then use the methods of HiLdEBRANDT to arrive at the result of JAKIMOVSKI and ENDL. This method enables us also to give the solution of the moment problems of the type $\mu_{n}=\int_{0}^{1} t^{n+\alpha} f(t) d t$ where $f$ is in any of the function spaces discussed by LoRENTZ [8]. The uniform approximation theorem in question is itself preceded by and derived from a general theorem on uniform approximation, which in turn is a generalization of a known theorem of Borman [1].

\section{Theorems on uniform approximation}

We start with the following general theorem on the uniform approximation of continuous functions. This theorem is an extension of a known theorem of BoHmaN [I] who considered the case when the $c_{m n}(x)$ are all positive, for each $m$ and $n$ and for $x$ in $[a, b]$ and $[c, d] \equiv[a, b]$.

Theorem 1. For given $a$ and $b,-\infty<a<b<\infty$ and for $0 \leqq n \leqq n(m)$, let $\left\{a_{m n}\right\}$ be defined, with

$$
c \leqq a_{m, 0}<a_{m, 1}<\cdots<a_{m, n(m)} \leqq d \quad \text { where } \quad[a, b] \subset[c, d] .
$$

Let the functions $c_{m n}(x)$ exist for $0 \leqq n \leqq n(m)$ and be finite for $a \leqq x \leqq b$. Suppose that for some $n_{0}, c_{m n}(x) \geqq 0$ for $n_{0} \leqq n \leqq n(m)$ and $x \in[a, b]$. Assume also that the following conditions hold, uniformly in $x$, for $a \leqq x \leqq b$ :

$$
\begin{gathered}
\lim _{m \rightarrow \infty} c_{m n}(x)=0, \text { for } n=0,1, \ldots, n_{0}-1, \\
\lim _{m \rightarrow \infty} \sum_{n=0}^{n(m)} c_{m n}(x)=1 \\
\lim _{m \rightarrow \infty} \sum_{n=0}^{n(m)} c_{m n}(x) a_{m n}=x \\
\lim _{m \rightarrow \infty} \sum_{n=0}^{n(m)} c_{m n}(x) a_{m n}^{2}=x^{2}
\end{gathered}
$$

Then

i) if $f(x)$ is bounded in $[c, d]$ and $x \in[a, b]$ is a point of continuity of $f(x)$ then

$$
\lim _{m \rightarrow \infty} \sum_{n=0}^{n(m)} c_{m n}(x) f\left(a_{m n}\right)=f(x) ;
$$


ii) if $f(x)$ is bounded in $[c, d]$ and is continuous in $a \leqq a^{*} \leqq x \leqq b^{*} \leqq b$, then we have, uniformly in $x$ for $x$ in $\left[a^{*}, b^{*}\right]$,

$$
\lim _{m \rightarrow \infty} \sum_{n=0}^{n(m)} c_{m n}(x) f\left(a_{m n}\right)=f(x) \text {. }
$$

Proof. We prove the conclusion (i); the proof of (ii) will easily follow from that.

First we make the following observations. Let $a \leqq x \leqq b$. Then

$$
\begin{aligned}
\lim _{m \rightarrow \infty} \sum_{n=0}^{n(m)}\left|c_{m n}(x)\right| & =\lim _{m \rightarrow \infty}\left\{\left|c_{m 0}(x)\right|+\left|c_{m 1}(x)\right|+\cdots+\left|c_{m, n_{0}-1}(x)\right|+\sum_{n=n_{0}}^{n(m)}\left|c_{m n}(x)\right|\right\} \\
& =\lim _{m \rightarrow \infty} \sum_{n=n_{0}}^{n(m)} c_{m n}(x)=\lim _{m \rightarrow \infty} \sum_{n=0}^{n(m)} c_{m n}(x),
\end{aligned}
$$

since $\lim _{m \rightarrow \infty} c_{m n}(x)=0$, for $n=0,1, \ldots, n_{0}-1$ and $\left|c_{m n}(x)\right|=c_{m n}(x)$ for $n \geqq n_{0}$.

Thus

Similarly

and

$$
\lim _{m \rightarrow \infty} \sum_{n=0}^{n(m)}\left|c_{m n}(x)\right|=1
$$

$$
\lim _{m \rightarrow \infty} \sum_{n=0}^{n(m)}\left|c_{m n}(x)\right| a_{m n}=x
$$

$(4)^{\prime}$

$$
\lim _{m \rightarrow \infty} \sum_{n=0}^{n(m)}\left|c_{m n}(x)\right| a_{m n}^{2}=x^{2}
$$

all limits being uniform for $x$ in $[a, b]$.

We have, next, for $x$ in $[a, b]$

(5) $f(x)-\sum_{n=0}^{n(m)} c_{m n}(x) f\left(a_{m n}\right)=f(x)\left[1-\sum_{n=0}^{n(m)} c_{m n}(x)\right]+\sum_{n=0}^{n(m)} c_{m n}(x)\left[f(x)-f\left(a_{m n}\right)\right]$.

Let $x$ be a point of continuity of $f(x)$ in $[a, b]$; then (with the usual meaning for $\varepsilon$ and $\delta$ ) we have

$$
\begin{aligned}
& f(x)-\sum_{n=0}^{n(m)} c_{m n}(x) f\left(a_{m n}\right) \\
& =f(x)\left[1-\sum_{n=0}^{n(m)} c_{m n}(x)\right]+\sum_{n \in \Delta_{x}^{\prime}} c_{m n}(x)\left[f(x)-f\left(a_{m n}\right)\right]+\sum_{n \in \Delta_{x}} c_{m n}(x)\left[f(x)-f\left(a_{m n}\right)\right],
\end{aligned}
$$

(where $\Delta_{x}=$ the set of $n$ such that $\left|a_{m n}-x\right|>\delta$, and $\Delta_{x}^{\prime}$ its complement)

$$
=\sum_{1}+\sum_{2}+\sum_{3}, \quad \text { say. }
$$

Since $f(x)$ is bounded in $[c, d]$ we get from (2),

$$
\lim _{m \rightarrow \infty} \sum_{1}=0 \text {. }
$$


Considering $\sum_{2}$, we have,

$$
\left|\sum_{2}\right| \leqq \varepsilon \sum_{n=0}^{n(m)}\left|c_{m n}(x)\right| \rightarrow 0, \text { by }(2)^{\prime} .
$$

Also,

Thus

$$
\begin{aligned}
\left|\sum_{3}\right| & \leqq 2 M \sum_{n \in \Delta_{x}}\left|c_{m n}(x)\right|, \text { where }|f(x)| \leqq M, \\
& \leqq \frac{2 M}{\delta^{2}} \sum_{n \in \Delta_{x}}\left|c_{m n}(x)\right|\left(a_{m n}-x\right)^{2} \\
& \leqq \frac{2 M}{\delta^{2}} \sum_{n=0}^{n(m)}\left|c_{m n}(x)\right|\left(a_{m n}-x\right)^{2} \\
& \rightarrow 0, \quad \text { by }(2)^{\prime},(3)^{\prime} \text { and }(4)^{\prime} .
\end{aligned}
$$

$$
\limsup _{m \rightarrow \infty}\left|f(x)-\sum_{n=0}^{n(m)} c_{m n}(x) f\left(a_{m n}\right)\right|<3 \varepsilon,
$$

and letting $\varepsilon \rightarrow 0$ we get the conclusion in (i).

The proof of the theorem is now complete.

Before proceeding to our main result in Theorem 2, we collect in the lemma below some relevant details. In what follows the binomial coefficient $\left(\begin{array}{c}m+\alpha \\ m-n\end{array}\right), \alpha$ real and $m, n$ are integers, is to be interpreted as usual; (i.e.)

$$
\left(\begin{array}{c}
m+\alpha \\
m-n
\end{array}\right)=\frac{(m+\alpha)(m-1+\alpha) \ldots(n+1+\alpha)}{1 \cdot 2 \cdot \ldots \cdot(m-n)},
$$

whenever $\alpha$ is not a negative integer and if $\alpha$ is a negative integer then

and

$$
\left(\begin{array}{c}
m+\alpha \\
m-n
\end{array}\right)=0 \text { if } n<-\alpha ; \text { also }\left(\begin{array}{l}
\alpha \\
0
\end{array}\right)=1 \quad \text { for all } \alpha
$$

$$
\left(\begin{array}{c}
m+\alpha \\
m-n
\end{array}\right)=0 \quad \text { for } n>m \text {. }
$$

At this stage we recall also the definition of the $\left(H^{\alpha}, \mu\right)$ transformation of a sequence defined by ENDL [2]. The $\left(H^{\alpha}, \mu\right)$ transformation is provided by the matrix $\left(H^{\alpha}, \mu\right) \equiv\left(h_{m n}^{\alpha}\right)$ where

$$
h_{m n}^{\alpha}=\left(\begin{array}{c}
m+\alpha \\
m-n
\end{array}\right) \Delta^{m-n} \mu_{n}, \quad(m \geqq n) \quad \text { and } \quad=0, \quad(m<n) .
$$

Thus the transform $\left(t_{n}\right)$ of a sequence $\left(s_{n}\right)$ by the $\left(H^{\alpha}, \mu\right)$ method will be defined by

$$
t_{m} \equiv h_{m}^{\alpha}\left\{s_{n} ; \mu_{n}\right\}=\sum_{n=0}^{m}\left(\begin{array}{c}
m+\alpha \\
m-n
\end{array}\right) \Delta^{m-n} \mu_{n} \cdot s_{n} .
$$


Lemma. The following equations are true when $\alpha \geqq 0,0 \leqq x \leqq 1$, or $\alpha=$ $-1,-2,-3, \ldots, 0 \leqq x \leqq 1$ or $\alpha<0, \alpha \neq-1,-2, \ldots$ and $0<x \leqq 1$ :

$$
\begin{aligned}
& \sum_{n=0}^{m}\left(\begin{array}{c}
m+\alpha \\
m-n
\end{array}\right) x^{n+\alpha}(1-x)^{m-n}=x^{\alpha} \sum_{n=0}^{m}\left(\begin{array}{c}
n+\alpha-1 \\
n
\end{array}\right)(1-x)^{n} ; \\
& \sum_{n=0}^{m}\left(\begin{array}{c}
m+\alpha \\
m-n
\end{array}\right) x^{n+\alpha}(1-x)^{m-n} \frac{n+\alpha}{m+\alpha}=x^{\alpha} \sum_{n=0}^{m}\left(\begin{array}{c}
n+\alpha-2 \\
n
\end{array}\right)(1-x)^{n} ; \\
& \sum_{n=0}^{m}\left(\begin{array}{c}
m+\alpha \\
m-n
\end{array}\right) x^{n+\alpha}(1-x)^{m-n} \frac{n+\alpha}{m+\alpha} \cdot \frac{n+\alpha-1}{m+\alpha-1}=x^{\alpha} \sum_{n=0}^{m}\left(\begin{array}{c}
n+\alpha-3 \\
n
\end{array}\right)(1-x)^{n} .
\end{aligned}
$$

Proof of the lemma. It has been shown by ENDL [2] that the product of $\left(H^{\alpha}, \mu\right)$ transformations is commutative; (i.e.)

Consider

$$
\left(H^{\alpha}, \mu\right)\left[\left(H^{\alpha}, v\right) s\right]=\left(H^{\alpha}, v\right)\left[\left(H^{\alpha}, \mu\right) s\right]=\left(H^{\alpha}, \mu v\right) s .
$$

then

$$
v_{n}=n+\alpha, \quad(n \geqq 0) ; \quad \text { if } \quad t_{m}=\left[\left(H^{\alpha}, v\right) s\right]_{m},
$$

$$
t_{0}=\alpha s_{0} \quad \text { and } \quad t_{m}=(m+\alpha)\left(s_{m}-s_{m-1}\right), \quad(m \geqq 1) .
$$

The choice $s_{n} \equiv 1$ now gives, by commutativity of the transformations, that for $m>0$

Thus

$$
\begin{gathered}
(m+\alpha)\left\{\sum_{n=0}^{m}\left(\begin{array}{c}
m+\alpha \\
m-n
\end{array}\right) \Delta^{m-n} \mu_{n}-\sum_{n=0}^{m-1}\left(\begin{array}{c}
m-1+\alpha \\
m-1-n
\end{array}\right) \Delta^{m-1-n} \mu_{n}\right\} \\
=\sum_{n=0}^{m}\left(\begin{array}{c}
m+\alpha \\
m-n
\end{array}\right) \Delta^{m-n} \mu_{n}\left[(n+\alpha)\left(s_{n}-s_{n-1}\right)\right] \\
=\left(\begin{array}{c}
m+\alpha \\
m
\end{array}\right) \Delta^{m} \mu_{0} \cdot \alpha .
\end{gathered}
$$

$$
\sum_{n=0}^{m}\left(\begin{array}{c}
m+\alpha \\
m-n
\end{array}\right) \Delta^{m-n} \mu_{n}=\sum_{n=0}^{m}\left(\frac{\alpha}{n+\alpha}\right)\left(\begin{array}{c}
n+\alpha \\
n
\end{array}\right) \Delta^{n} \mu_{0}=\sum_{n=0}^{m}\left(\begin{array}{c}
n+\alpha-1 \\
n
\end{array}\right) \Delta^{n} \mu_{0} .
$$

Choose now $\mu_{n}=x^{n+\alpha},(n \geqq 0)$; then we get (7). Since

$$
\left(\begin{array}{l}
m+\alpha \\
m-n
\end{array}\right)\left(\frac{n+\alpha}{m+\alpha}\right)=\left(\begin{array}{c}
m+\alpha-1 \\
m-n
\end{array}\right)
$$

we obtain (8) from (7). Similarly (9) can be obtained from (7) or (8).

Remark. In case $\alpha$ is a negative integer then the sums on the right side of each of the Eqs. (7) through (9) are finite sums and therefore, for $m \geqq m_{0}(\alpha)$ they remain (respectively) unchanged and thus for $m \geqq m_{0}(\alpha)$ each one of the sums on the left side remains unchanged.

We prove now

Theorem 2. Let $\alpha$ be a real number. Let $f(x)$ be bounded in $\beta \leqq x \leqq 1$, where

$$
\beta=\inf \left(\frac{n+\alpha}{m+\alpha}\right) .
$$


Let

$$
B_{m}(\alpha, f, x)=\sum_{n=0}^{m}\left(\begin{array}{c}
m+\alpha \\
m-n
\end{array}\right)(1-x)^{m-n} x^{n+\alpha} f\left(\frac{n+\alpha}{m+\alpha}\right), \quad m=0,1,2, \ldots
$$

Then

i) at each point of continuity of $f(x)$ in $0<x \leqq 1, B_{m}(\alpha, f, x)$ tends to $f(x)$;

ii) if $f(x)$ is continuous in some interval $0<a \leqq x \leqq b \leqq 1$, then $B_{m}(\alpha, f, x)$ converges to $f(x)$ uniformly in $a \leqq x \leqq b$;

iii) if $\alpha$ is a non-positive integer then in (i) and (ii) above, the $<$ symbol following 0 can be replaced by $\leqq$.

Proof of the theorem. If $-p \geqq \alpha>-(p+1)$ where $p$ is a positive integer, then

If

$$
\left(\begin{array}{c}
m+\alpha \\
m-n
\end{array}\right) \geqq 0 \text { for } \quad p \leqq n \leqq m=p, p+1, \ldots
$$

$$
\alpha=-p, \text { then }\left(\begin{array}{l}
m+\alpha \\
m-n
\end{array}\right)=0 \text { for } 0 \leqq n<p \leqq m .
$$

Also, it can be easily seen that for each fixed $n \geqq 0$, we have, uniformly in $x$ for $0<\delta \leqq x \leqq 1$,

$$
\lim _{m \rightarrow \infty}\left(\begin{array}{l}
m+\alpha \\
m-n
\end{array}\right)(1-x)^{m-n} x^{n+\alpha}=0 .
$$

From our lemma, we have [on evaluating the sums on the right side of Eq. (7), (8) and (9)] that the following equations hold, uniformly in $x$, for $0<\delta \leqq x \leqq 1$ :

Since

$$
\begin{aligned}
& \lim _{m \rightarrow \infty} \sum_{n=0}^{m}\left(\begin{array}{l}
m+\alpha \\
m-n
\end{array}\right)(1-x)^{m-n} x^{n+\alpha}=1, \\
& \lim _{m \rightarrow \infty} \sum_{n=0}^{m}\left(\begin{array}{l}
m+\alpha \\
m-n
\end{array}\right)(1-x)^{m-n} x^{n+\alpha} \cdot \frac{n+\alpha}{m+\alpha}=x, \\
& \lim _{m \rightarrow \infty} \sum_{n=0}^{m}\left(\begin{array}{l}
m+\alpha \\
m-n
\end{array}\right)(1-x)^{m-n} x^{n+\alpha} \cdot \frac{n+\alpha}{m+\alpha} \cdot \frac{n+\alpha-1}{m+\alpha-1}=x^{2} .
\end{aligned}
$$

$$
\begin{aligned}
\left(\frac{n+\alpha}{m+\alpha}\right)^{2}-\frac{n+\alpha}{m+\alpha} \cdot \frac{n+\alpha-1}{m+\alpha-1} & =\frac{n+\alpha}{m+\alpha} \cdot \frac{m-n}{(m+\alpha)(m+\alpha-1)} \\
& \leqq \frac{n+\alpha}{m+\alpha} \cdot \frac{m}{(m+\alpha)(m+\alpha-1)}
\end{aligned}
$$

we have, by (12), (13) and (14) that

$$
\lim _{m \rightarrow \infty} \sum_{n=0}^{m}\left(\begin{array}{c}
m+\alpha \\
m-n
\end{array}\right)(1-x)^{m-n} x^{n+\alpha}\left(\frac{n+\alpha}{m+\alpha}\right)^{2}=x^{2} .
$$

Now the theorem follows from Theorem 1 by utilising (11), (12), (13) and (15) and also the remark following the lemma. 
Note. If $\alpha$ is non-negative then $\beta=0$; for $\alpha=0$, we get the Bernstein approximation theorem.

\section{Applications}

We give now the application of our Theorem 2 to the solution of certain moment problems. We start with a sequence $\left\{\mu_{n}\right\}$ of real constants and shall be concerned with the determination of the necessary and sufficient conditions, involving $\mu_{n}$, so that for each $n, \mu_{n}$ has either of the following representations:

$$
\mu_{n}=\int_{0}^{1} t^{n+\alpha} d \chi(t)
$$

where $\chi$ is a function of bounded variation over the interval $[0,1]$ or

$$
\mu_{n}=\int_{0}^{1} t^{n+\alpha} f(t) d t,
$$

where $f(t)$ belongs to a suitable function space like the space $L^{p}[0,1]$ or more generally to the space $X(C)$ of LoRENTZ [8] whose definition is found elsewhere in this section.

First we shall deal with the representation of $\mu_{n}$ in the form (16) and prove the following theorem (Theorem 3), one alternate proof of which is due to ENDL [2] and another is due to JAKIMOVSKI [7]. We remark here that we shall be content with proving Theorem 3 for $\alpha>0$ and refer the reader to the papers by ENDL and by JAKIMOVSKI, where the case $\alpha<0$ is discussed and the discussion is based on the case of positive $\alpha$.

Theorem $3^{1}$ ). Let $\alpha>0$. The real sequence $\mu_{n}$ has the representation (16) where $\chi$ is a function of bounded variation over $[0,1]$ if and only if

$$
\sup _{m} \sum_{n=0}^{m}\left(\begin{array}{c}
m+\alpha \\
m-n
\end{array}\right)\left|\Delta^{m-n} \mu_{n}\right|<\infty .
$$

Proof of the sufficiency. We start with the assumption that

$$
\sup _{m} \sum_{n=0}^{m}\left(\begin{array}{c}
m+\alpha \\
m-n
\end{array}\right)\left|\Delta^{m-n} \mu_{n}\right|=N<\infty .
$$

Consider the linear space $Q$ of polynomials of (possibly) fractional powers, of the type

$$
q(x)=a_{0} x^{\alpha}+a_{1} x^{1+\alpha}+\cdots+a_{k} x^{k+\alpha}
$$

and $x \in[\delta, 1], \delta>0$.

Consider also the linear space $P$ of polynomials of the type

$$
p(x)=a_{0}+a_{1} x+\cdots+a_{k} x^{k},
$$

for $x$ in the same interval as before.

1) The case $\alpha=1$ has been discussed by RamanuJan [11]. 
We can associate in a 1-1 manner, to a polynomial $q(x)$ in $Q$ a polynomial, to be precise the polynomial $p(x)$, in $P$. This can be easily shown to be a linear homeomorphism between the Banach spaces $P$ and $Q$, each with its natural supremum norm topology. We shall indeed have

$$
\|q\| \leqq\|p\| \leqq \frac{1}{\delta^{x}}\|q\|
$$

We denote now by $L(p)$ as also by $\bar{L}(q)$ the quantity $a_{0} \mu_{0}+a_{1} \mu_{1}+\cdots+a_{k} \mu_{k}$. Since for

$$
\alpha>0, \quad\left(\begin{array}{c}
m \\
n
\end{array}\right) \leqq\left(\begin{array}{c}
m+\alpha \\
m-n
\end{array}\right) \quad \text { for all } m \text { and } n,
$$

we have in virtue of our hypothesis that

$$
\sum_{n=0}^{m}\left(\begin{array}{l}
m \\
n
\end{array}\right)\left|\Delta^{m-n} \mu_{n}\right| \leqq N \quad \text { for all } m
$$

But, whenever the condition (21) above is satisfied, it can be shown, as has been demonstrated by LoRENTZ ( $[8]$, p. $58-59)$ that $L(p)$ is a linear continuous functional on $P$ and that $|L(p)| \leqq N\|p\|$ where $N$ is the same for all $p \in P$. Thus

$$
|\bar{L}(q)|=|L(p)| \leqq N\|p\| \leqq N \cdot \frac{1}{\delta^{\alpha}}\|q\|=\bar{M}\|q\|, \quad \text { for all } q \in Q .
$$

Consequently $\bar{L}(q)$ is a linear continuous functional over $Q$.

Consider now the member $q_{m}^{k+\alpha}(x)$ of $Q$, defined by

Then

$$
q_{m}^{k+\alpha}(x) \equiv B_{m}\left(\alpha, x^{k+\alpha}, x\right)=\sum_{n=0}^{m}\left(\begin{array}{c}
m+\alpha \\
m-n
\end{array}\right)(1-x)^{m-n} x^{n+\alpha}\left(\frac{n+\alpha}{m+\alpha}\right)^{k+\alpha} \text {. }
$$

$$
\bar{L}\left(q_{m}^{k+\alpha}\right)=\sum_{n=0}^{m}\left(\begin{array}{c}
m+\alpha \\
m-n
\end{array}\right) \Delta^{m-n} \mu_{n} \cdot\left(\frac{n+\alpha}{m+\alpha}\right)^{k+\alpha} .
$$

By Theorem 2, $q_{m}^{k+\alpha}(x)$ converges uniformly, and therefore in norm, to $x^{k+\alpha}$. Since $q_{m}^{k+\alpha}(x), m=0,1, \ldots$ are all members of $Q$ and $\bar{L}$ is a linear continuous functional over $Q$, we have $\bar{L}\left(q_{m}^{k+\infty}\right) \rightarrow \bar{L}\left(x^{k+\alpha}\right)$ as $m \rightarrow \infty$; i.e.

$$
\lim _{m \rightarrow \infty} \sum_{n=0}^{m}\left(\begin{array}{c}
m+\alpha \\
m-n
\end{array}\right) \Delta^{m-n} \mu_{n} \cdot\left(\frac{n+\alpha}{m+\alpha}\right)^{k+\alpha}=\mu_{k} .
$$

We shall now define a sequence of functions $\chi_{m}(t)$ as follows: for each fixed $m$, $\chi_{m}(0)=0$ and it is a step function with the jumps

$$
\left(\begin{array}{c}
m+\alpha \\
m-n
\end{array}\right) \Delta^{m-n} \mu_{n} \text { at } t=\frac{n+\alpha}{m+\alpha}, \quad n=0,1,2, \ldots, m .
$$

$\left.{ }^{2}\right)$ The above equation is very similar to the one obtained by JAKIMOvSKI ([7], p. 30); however the above equation can also be obtained, following the method of JAKIMOVSKI [6] and utilising the equation (11.13) of [7] and in this case the above equation can indeed be obtained with no restriction on $\mu_{n}$. 
Thus for each $m, \chi_{m}(t)$ is a function of bounded variation in $[0,1]$, since by hypothesis

Also, Eq. (22) gives that

$$
\sum_{n=0}^{m}\left(\begin{array}{c}
m+\alpha \\
m-n
\end{array}\right)\left|\Delta^{m-n} \mu_{n}\right| \leqq N .
$$

$$
\mu_{k}=\lim _{m \rightarrow \infty} \int_{0}^{1} t^{k+\alpha} d \chi_{m}(t), \quad k=0,1,2,3, \ldots .
$$

Now since the functions $\chi_{m}(t)$ are all of uniform-bounded-variation first an application of the Helly theorem (see WIDDER [14], p. 29) and then of the Helly-Bray theorem (WrDDER [14], p. 31) yields a function $\chi(t)$ of variation bounded by $N$ and such that

$$
\mu_{k}=\int_{0}^{1} t^{k+\alpha} d \chi(t), \quad k=0,1,2, \ldots
$$

Thus the condition is sufficient.

That the condition is necessary follows from the following

Lemma. For $0 \leqq t \leqq 1$ and $\alpha>0$,

$$
\sum_{n=0}^{m}\left(\begin{array}{c}
m+\alpha \\
n
\end{array}\right)(1-t)^{n} t^{m+\alpha-n} \leqq 1 .
$$

The proof of the above lemma follows from Eq. (4.2.4) of ENDL [2].

We now indicate briefly the method of solution of the moment problem (17) i.e. to represent the sequence $\left(\mu_{n}\right)$ in the form

$$
\mu_{n}=\int_{0}^{1} t^{n+\alpha} f(t) d t,
$$

where $f$ belongs to the space $X(C)$ defined as below:

The class $C$ is a class of positive integrable functions defined on $[0,1]$ and the class possesses the properties

1) $1 \in C$,

2) the class $C$ is normal in the sense that if $c_{1}(x) \in C$ and $c_{2}(x) \leqq c_{1}(x)$ a.e. then $c_{2}(x) \in C$,

3) the integrals $\int_{0}^{1} c(x) d x, c \in C$, are bounded.

The space $X(C)$ consists of all measurable functions $f$ for which

$$
\sup _{c \in C} \int_{0}^{1}|f(x)| c(x) d x=\|f\|<\infty .
$$

That the space $X(C)$ is a Banach space, with the norm $\|f\|$ is easily verified; also suitable choice of the class $C$ will yield $L^{p}(p>1)$ and also the Orlicz spaces (for details on this, see, LORENTZ, pp. 65-68). 
Define now the functions $f_{m}^{\alpha}(x)$ and $K_{m}^{\alpha}(x, t)$ as follows:

$$
\begin{aligned}
& f_{m}^{\alpha}(x)=(m+\alpha+1)\left(\begin{array}{l}
m+\alpha \\
m-n
\end{array}\right) \Delta^{m-n} \mu_{n}, \quad \mid \frac{n+\alpha}{m+\alpha+1} \leqq x<\frac{n+\alpha+1}{m+\alpha+1}, \\
& \left.K_{m}^{\alpha}(x, t)=(m+\alpha+1)\left(\begin{array}{c}
m+\alpha \\
m-n
\end{array}\right)(1-t)^{m-n} t^{n+\alpha}\right\} \begin{array}{c}
n=0,1,2, \ldots, m ; \\
m=0,1,2, \ldots .
\end{array} \\
& \left.\begin{array}{r}
f_{m}^{\alpha}(x)=0 \\
K_{m}^{\alpha}(x, t)=0
\end{array}\right\} 0 \leqq x<\frac{\alpha}{m+\alpha+1}, \quad m=0,1,2, \ldots
\end{aligned}
$$

It is then easily verified that

as also

$$
\int_{0}^{1} K_{m}^{\alpha}(x, t) d t=1
$$

$$
\int_{0}^{1} K_{m}^{\alpha}(x, t) d x=\sum_{n=0}^{m}\left(\begin{array}{c}
m+\alpha \\
m-n
\end{array}\right)(1-t)^{m-n} t^{n+\alpha} \leqq 1
$$

in virtue of lemma above.

Further, if

$$
\mu_{m}=\int_{0}^{1} t^{m+\alpha} f(t) d t, \text { then } f_{m}^{\alpha}(x)=\int_{0}^{1} K_{m}^{\alpha}(x, t) f(t) d .
$$

The above facts, and Eq. (22) lead to the following theorem, the proof of which is parallel to the proof of the same result in case $\alpha=0$ given earlier by LORENTZ [8].

Theorem 4. Let the space $X(C)$ have the property of re-arrangement invariant norm and let the integrals

$$
\int_{0}^{1} f(x) d x, \quad f \in X \quad \text { and } \quad\|f\| \leqq 1
$$

have the property of uniform absolute continuity. Then for $\alpha>0$ the real sequence $\left(\mu_{n}\right)$ has the representation

$$
\mu_{n}=\int_{0}^{1} t^{n+\alpha} f(t) d t
$$

with $f \in X(C)$ and with $\|f\| \leqq M$, if and only if for each $m,\left\|f_{m}^{\alpha}\right\| \leqq M$.

At this stage we make the following observations. A known theorem of MEYER-KöNIG and ZELLER [9] gives the following result.

Theorem 5. Let $f(x)$ be continuous in $[\delta, 1], \delta>0$, and let for $\alpha>0$ the sequence of functions $P_{m}(\alpha, f, x)$ be defined by

$$
P_{m}(\alpha, f, x)=\sum_{n=m}^{\infty}\left(\begin{array}{l}
n+\alpha \\
n-m
\end{array}\right)(1-x)^{n-m} x^{m+\alpha+1} f\left(\frac{m+\alpha+1}{n+\alpha+1}\right) .
$$

Then $P_{m}(\alpha, f, x)$ uniformly approximate $f(x)$ for $x$ in $[\delta, 1]$.

Utilising Theorem 5 in the place of Theorem 2 we can prove the following analogues of Theorems 3 and 4. 
Theorem $6^{3}$ ). A real sequence $\left(\mu_{n}\right)$ has the representation in Eq. (16) with the function $\chi(t)$ being of bounded variation in $[0,1]$ if and only if

$$
\sup _{m} \sum_{n=m}^{\infty}\left(\begin{array}{l}
n+\alpha \\
n-m
\end{array}\right)\left|\Delta^{n-m} \mu_{m+1}\right|<\infty \text {. }
$$

Theorem 7. Let the sequence of functions $F_{m}^{\alpha}(x)$ be defined as follows:

$F_{m}^{\alpha}(x)=\frac{(n+\alpha+1)(n+\alpha+2)}{(m+\alpha+1)}\left(\begin{array}{c}n+\alpha \\ n-m\end{array}\right) \Delta^{n-m} \mu_{m+1},\left\{\begin{array}{l}\frac{m+\alpha+1}{n+\alpha+2}<x \leqq \frac{m+\alpha+1}{n+\alpha+1}, \\ m=0,1,2, \ldots, \\ n=m, m+1, \ldots\end{array}\right.$

Then the real sequence $\left\{\mu_{n}\right\}$ has the representation (17) with $f \in X(C)$, where the space $X(C)$ satisfies the hypothesis in Theorem 4 , and with $\|f\| \leqq M$, if and only if, for each $m,\left\|F_{m}^{\alpha}\right\| \leqq M$.

In the case $\alpha=0$ the result is due to RAMANUJAN [12] and the proof of the present theorem is obtained on similar lines.

\section{References}

[1] Bohman, H.: On approximation of continuous and of analytic functions. Ark. Mat. 2, 43- 56 (1952).

[2] ENDL, K.: Untersuchungen über Momentenprobleme bei Verfahren vom Hausdorffschen Typus. Math. Ann. 139, 403-432 (1960).

[3] Hardy, G. H.: Divergent Series. Oxford: Clarendon 1949.

[4] HausdorfF, F.: Summationsmethoden und Momentfolgen, I, II. Math. Z. 9, 74-109; $280-299$ (1921).

[5] Hildebrandt, T.H.: On the moment problem for a finite interval. Bull. Amer. math. Soc. 38, 269-270 (1932).

[6] JAKIMOvskI, A.: Some remarks on the moment problem of Hausdorff. J. London math. Soc. 33, 1-14 (1958).

[7] - The product of summability methods; part 2. Technical report no. 8, Jerusalem 1959.

[8] LoRentz, G.G.: Bernstein polynomials. Toronto: University of Toronto Press 1953.

[9] MeYer-KöNIG, W., and K. Zeller: Bernsteinsche Potenzreihen. Studia Math. 19, 89-94 (1960).

[10] Ramanujan, M.S.: Series-to-series quasi-Hausdorff transformations. J. Indian math. Soc. 17, 47-53 (1953).

[11] - On Hausdorff and quasi-Hausdorff methods of summability. Quart. J. Math. (Oxford II) 8, 197-213 (1957).

[12] - The moment problem in a certain function space of G.G. Lorentz. Archiv der Math. 15, 71-75 (1964).

[13] Shohat, J.A., and J.D. Tamarkin: The problem of moments. Providence: Amer. math. Soc. 1943.

[14] Widder, D.V.: The Laplace transform. Princeton: Princeton University Press 1941.

The University of Tel-Aviv, Tel-Aviv, Israel University of Michigan, Ann Arbor, Mich., U.S.A and Ramanujan Institute of Mathematics, Madras, India

(Received October 5, 1963)

\footnotetext{
${ }^{3}$ ) An alternate proof to Theorem 6, based on an extended version of Theorem 3, has been given by JAKIMOVSKI [7].
} 